\title{
Ulnar osteotomy is the only required procedure for chronic monteggia fracture in children.
}

1. MBBS, FCPS

Assistant Professor Paediatric

Orthopaedics

The Children's Hospital and

The Institute of Child Health, Lahore

2. MBBS, FCPS

Senior Registrar Paediatric

Orthopaedics

The Children's Hospital and

The Institute of Child Health, Lahore.

3. MBBS, FCPS

Senior Registrar Paediatric

Orthopaedics

The Children's Hospital and

The Institute of Child Child Health,

Lahore.

4. MBBS, FCPS

Professor Paediatric Orthopaedics

The Children's Hospital and

The Institute of Child Health, Lahore.

Correspondence Address:

Dr. Abdul Latif Shahid

Department of Paediatric

Orthopaedics,

The Children's Hospital and

The Institute of Child Health, Lahore.

abdullatifshahid01@gmail.com

Article received on:

03/09/2020

Accepted for publication:

02/03/2021

\begin{abstract}
Abdul Latif Shahid', Farhad Alam ${ }^{2}$, Islam Hussain ${ }^{3}$, Abdul Latif Sami ${ }^{4}$
\end{abstract}
ABSTRACT... Objective: To determine containment of radial head after ulnar osteotomy in chronic Monteggia fractures. Study Design: Retrospective study. Setting: Children Hospital and The Institute of Child Health, Lahore. Period: 2019 to January 2020. Material \& Methods: Ten patients presented in outdoor patient department with a diagnosis of chronic Monteggia fracture. Four patients were labelled as missed Monteggia fractures, four with late presented Monteggia fractures and two with inadequately treated Monteggia fractures. Bado and Letts classifications were applied for patients. Mean age was 6 years and 8 months and ranged from 4 to 10 years. Mean time interval between injury and admission was 6.1 months. Open reduction of radial head and ulnar osteotomy was done through Boyed approach. Transcapitellar wire was inserted temporarily and then removed so it is not required permanently. The ulnar osteotomy was angulated opposite to the direction of radial head dislocation and fixed with plate and screws. Results: 10 patients were included in this study. The age range was between 4 and 10 years. The study period was six months and follow up was one year. Mean ulnar angulation at osteotomy site was $21.3^{\circ}\left(16-25^{\circ}\right)$. Mean ulnar lengthening at osteotomy site was $0.85 \mathrm{~cm}(0.5-1.8 \mathrm{~cm})$. Improvement in flexion-extension was $20.3 \%$, pronation was $5.1 \%$ and supination was $13.7 \%$. Complications included were nounion in 1 case and cubitus valgus in 1 case. Conclusion: Containment of radial head is obtained by open reduction of radial head and with ulnar osteotomy in chronic Monteggia fractures. Annular ligament reconstruction and transcapitellar wire insertion are not required if proper angulation and fixation of ulnar osteotomy is performed. No age limit for this procedure but surgery should be performed before radial head deformation.

Key words: Chronic Monteggia Fracture, Containment, Ulnar Osteotomy.

Article Citation: Shahid AL, Alam F, Hussain I, Sami AL. Ulnar osteotomy is the only required procedure for chronic monteggia fracture in children. Professional Med $\mathrm{J}$ 2021; 28(6):886-890. https://doi.org/10.29309/TPMJ/2021.28.06.6054

\section{INTRODUCTION}

Radial head dislocation with associated ulnar shaft fracture is called Monteggia fracture.

There are four types of Monteggia fractures according to Bado classification which is based on direction of radial head dislocation. Anterior, posterior and lateral radial head dislocations with ulnar shaft fractures are type I,II and III Monteggia fractures while anterior dislocation of radial head with both radial and ulnar shaft fractures is type IV. ${ }^{1}$ Letts classification is especially useful for pediatric population. There is anterior dislocation of radial head with plastic deformation of ulna, greenstick fracture of ulna and complete fracture of ulna in type $A, B$ and $C$ respectively. In type $D$, there is posterior dislocation of radial head with ulnar shaft fracture while there is lateral dislocation of radial head with ulnar shaft fracture in type $E{ }^{2}$

Chronic Monteggia fracture is a term used for missed Monteggia fracture, inadequately treated Monteggia fracture and late presented Monteggia fracture. ${ }^{3}$

Treatment of acute Monteggia fracture is very easy and usually close reduction of radial head is possible by adequately managing the ulnar shaft fracture because ulnar fracture geometry helps in stabilization. ${ }^{4}$

Chronic Monteggia fracture treatment, on the other hand, is very challenging and has many problems. The main difference of treatment between acute and chronic Monteggia fracture is 
the concept of reconstruction required for chronic cases and only fracture management needed for acute fractures.

There are different treatment strategies for chronic Monteggia fractures. Clearing the radial bed for reduction of radial head, ulnar osteotomy and angulation, annular ligament reconstruction and transcapitellar wire insertion are key steps for this chronic lesion. ${ }^{5,6,7,8}$

The ulnar osteotomy is the key procedure required for proper containment of radial head in its bed in chronic Monteggia fracture..$^{9,10}$ Annular ligament reconstruction required in this chronic lesion is debatable. ${ }^{11,12}$

The rationale of our study is to achieve containment of radial head by properly performed ulnar osteotomy in chronic Monteggia fractures.

\section{MATERIAL \& METHODS}

After approval from the ethical review committee of our hospital (Ref:46549). This descriptive case series study was done. Our sampling technique was non-probability, consecutive type. 10 patients who came in outpatient department of pediatric orthopedics of the Children Hospital and the Institute of Child Health, Lahore from January 2019 to January 2020, and fulfill the inclusion criteria were enrolled in the study. Patients aged 4-10 years with Monteggia fractures missed initially or not treated adequately initially or presented late were included in this study. All patients with open fractures on clinical examination (skin wound) and neurovascular injury on clinical examination (absent pulses and sensations distally) and multiple fractures were excluded from the study. Informed consent and demographic profile (name, age, sex, and contact) was obtained. The procedure details, benefits and complications explained to parents. The deformity was corrected via Boyed approach. First of all radial bed was prepared for containment of radial head. All fibrous tissue was removed from radial bed and then ulnar osteotomy was performed. Transcapitellar wire thru capitulum into radial head, neck and shaft was inserted temporarily and removed when ulnar osteotomy was fixed with screws and plate after giving proper angulation opposite to original direction of radial head dislocation. Finally, stability of radial head was checked by flexion and extension of elbow and supination and pronation of forearm. We applied above elbow cast.

On first postoperative day, detail neurovascular examination was done and both anteroposterior and lateral radiographs were taken. Patients were discharged on second postoperative day. Patients were called after two weeks for removal of stitches and after four weeks for removal of above elbow cast. Physiotherapy in form of elbow flexion and extension and forearm supination and pronation was also started on second visit. Then patients were called monthly for three months and quarterly until union achieved and full range of movement obtained. A single surgical team carried out all operations. Screws and plate was removed after union achieved.

The data was analyzed by using software SPSS version 17.0.The variables under study were age, gender, side and mechanism of injury. The variables were analyzed through simple, discriptive statistics. Numerical variables like age were presented by mean and standard deviation. Qualitative variables like gender, side and mechanism of injury were represented as frequency and percentage. Chi square was applied for post-stratification for age, malnutrition and gender. $\mathrm{P}$ value $\leq 0.05$ was considered significant.

\section{RESULTS}

Ten patients were included in this study. The age range was 4-10 years (mean age was 6 years and 8 months). The study period was six months and follow up was one year. The ulnar osteotomy and angulation was key step for success of this procedure, so mean ulnar angulation at osteotomy site was $21.3^{\circ}\left(16-25^{\circ}\right)$. With ulnar angulation, ulnar lengthening was achieved at osteotomy site and mean lengthening was $0.85 \mathrm{~cm}(0.5-1.8 \mathrm{~cm})$. There was improvement in elbow and forearm range of movements. Improvement in flexionextention was $20.3 \%$, pronation was $5.1 \%$ and supination was $13.7 \%$. There was no radial head 
dislocation or subluxation. On the other hand, there were two main complications included nounion in one case and cubitus valgus in other case. Bone grafting was done for nonunion and distal humeral osteotomy was done for cubitus valgus.

\section{DISCUSSION}

Monteggia fracture is a two forearm bones lesion including ulnar shaft fracture and radial head dislocation.

Proper diagnosis of Monteggia fracture is still a common problem. Whenever shaft of forearm bones is fractured, always include elbow in radiographs so that radial head dislocation may not be missed.

Direction of radial head dislocation is the main determinant of Bado classification.

Anterior, posterior and lateral radial head dislocations with ulnar shaft fractures are type I,II and III Monteggia fractures while anterior dislocation of radial head with both radial and ulnar shaft fractures is type IV ${ }^{1}$. Bado classification is more useful for adults. For pediatric population, Letts classification is used to describe this unique lesion. There are five types of pediatric Monteggia fracures. There are three types for anterior dislocation of radial head, one type for posterior dislocation and one type for lateral dislocation. In type $A$, there is plastic deformation of ulna, in type B greenstick fracture of ulna and in type $\mathrm{C}$ complete fracture of ulna along with anterior dislocation of radial head. In type D, there is posterior dislocation of radial head with ulnar shaft fracture while there is lateral dislocation of radial head with ulnar shaft fracture in type $\mathrm{E}^{2}$.

Treatment of acute Monteggia fracture is very easy and usually close reduction of radial head possible when adequately ulnar shaft fracture is managed because ulnar fracture geometry helps in stabilization. ${ }^{4}$ Chronic Monteggia fracture treatment, on the other hand, is very challenging and has many problems. The main difference of treatment between acute and chronic Monteggia fracture is the concept of reconstruction required for chronic cases and only fracture management needed for acute cases.

The ulnar osteotomy is the key procedure required for proper containment of radial head in its bed. ${ }^{9,10}$ This is the procedure we adopted in our this case series. The radial head is pushed anteriorly, posteriorly or laterally depending upon the apex of ulnar fracture. In ulnar osteotomy, the deformity is reversed so that radial head is rested into the radial bead. Radial head is kept reduced by creating tension in the interosseous membrane. Lengthening achieved by angulation of the osteotomy also provides more space for radial head in its anatomical bead. Hence, rationale of our osteotomy is three folds: creation of deformity opposite to direction of radial head dislocation, creation of tension in interosseous membrane and creation of lengthening by angulation of osteotomy. ${ }^{9,10}$

Annular ligament reconstruction required is debateable. ${ }^{11,12}$ Annular ligament reconstruction is always with complications like nerve injury, myositis ossificans and stiffness of elbow. ${ }^{13,14}$ Also, distraction and angulation at the osteotomy site pushes the radial head down through the interosseous membrane, eliminating for any ligament reconstruction requirement. ${ }^{15,16}$

There are two studies in which only ulnar osteotomy is done ${ }^{17,18}$ In study of Parkash, angulation and elongation of the ulna by ulnar osteotomy are required for reduction of the radial head and this is same in our study. In study of Park et al, ulnar osteotomy and correction of the ulnar deformity are the key components to achieve reduction and this is also true in our study Another study showed both ulnar ostetomy and annular ligament reconstruction are required. ${ }^{19}$ Another study shows good to excellent results in chronic Monteggia fractures if surgery performed before radial head is deformed. ${ }^{20}$

Transcapitellar wire insertion is also debatable. Transcapitellar wire can be used as a temporary fixation after reduction of radial head, as proposed by Bae. ${ }^{21}$ when ulna is fixed with screws and plate, transcapitellar wire removed. In all our cases we 
used this wire as a temporary fixation.

There are limitations in our study of this case series. Our follow-up is short and also control group is not present. In brief, open reduction of radial head and ulnar osteotomy with angulation opposite to direction of radial head dislocation is a good and applicable method in the treatment of chronic Monteggia fractures.

\section{CONCLUSION}

Containment of radial head is achieved by open reduction of radial head and ulnar osteotomy in chronic Monteggia fractures. Annular ligament reconstruction and transcapitellar wire insertion are not required if proper angulation and fixation of ulnar osteotomy is performed. No age limit for this procedure but surgery should be performed before radial head deformation.

Copyright@ 02 Mar, 2021.

\section{REFRENCES}

1. Bado JL. The Monteggia lesion. Clin Orthop Relat Res. $1967 ; 50: 71-86$.

2. Letts $M$, Locht $R$, Wiens J. Monteggia fracturedislocations in children. J Bone Joint Surg Br. 1985; 67:724-727.

3. Goyal T, Arora SS, Banerjee S, Kandwal P. Neglected Monteggia fracture dislocations in children: $A$ systematic review. J Pediatr Orthop Part B. 2015; 24(3):191-199.

4. Ramski DE, Hennrikus WP, Bae DS. Pediatric monteggia fractures: A multicenter examination of treatment strategy and early clinical and radiographic results. J Pediatr Orthop. 2015; 35: 115-120.

5. Gyr BM, Stevens PM, Smith JT. Chronic Monteggia fractures in children: outcome after treatment with the Bell-Tawse procedure. J Pediatr Orthop Part B. $2004 ; 13(6): 402-406$.

6. Eygendaal D, Hillen RJ. Open reduction and corrective ulnar osteotomy for missed radial head dislocations in children. Strateg Trauma Limb Reconstr. 2007; 2(1):31-34.

7. Belangero WD, Livani B, Zogaib RK. Treatment of chronic radial head dislocations in children. Int Orthop. 2007; 31(2):151-154.
8. Nakamura K, Hirachi K, Uchiyama S, Takahara M, Minami A, Imaeda $\mathrm{T}$, et al. Long-term clinical and radiographic outcomes after open reduction for missed Monteggia fracture-dislocations in children. $J$ Bone Joint Surg Am. 2009; 91(6):1394-1404.

9. Bor N, Rubin G, Rozen N, Herzenberg JE. Chronic anterior Monteggia lesions in children: report of 4 cases treated with closed reduction by ulnar osteotomy and external fixation. J Pediatr Orthop. 2015 Jan; 35(1):7-10.

10. BhaskarA. Missed Monteggia fracture in children: Is annular ligament reconstruction always required? Indian J Orthop. 2009 Oct; 43(4):389-95.

11. Hui JHP, Sulaiman AR, Lee H-C. Open reduction and annular ligament reconstruction with fascia of the forearm in chronic Monteggia lesions in children. $J$ Pediatr Orthop. 2005; 25:501-605.

12. Attarian DE. Annular ligament reconstruction in chronic posttraumatic radial head dislocation in children. Contemp Orthop. 1993; 27:259-264.

13. Rodgers WB, Waters PM, Hall JE. Chronic Monteggia lesions in children. Complications and results of reconstruction. J Bone Joint. 1996; 78-A:1322--1329.

14. De Boeck H. Radial neck osteolysis after annular ligament reconstruction. A case report. Clin Orthop Relat Res. 1997; 342:94-98.

15. Exner GU. Missed chronic anterior Monteggia lesion. Closed reduction by gradual lengthening and angulation of the ulna. $\mathrm{J}$ Bone Joint Surg. 2001; 83B:547--550.

16. Degreef I, De Smet L. Missed radial head dislocations in children associated with ulnar deformation: Treatment by open reduction and ulnar osteotomy. $J$ Orthop Trauma. 2004; 18: 375--378.

17. Chauhan $P$. Surgical treatment in children missed Monteggia lesions at Bhuj, Kutch, Gujarat: A retrospective study. Int J Res Orthop. 2017; 3(1): 3034.

18. Park H, Kwang-Won P, Park KB, Kim HW, Eom NK, and Lee $\mathrm{DH}$. Impact of open reduction on surgical strategies for missed monteggia fracture in children. Yonsei Med J 2017; 58(4): 829-836.

19. Lu X, Yan G, Wang Y, Zhu Z, You H, Zhang J, and Guo $Y$. Repositioning of the annular ligament in the management of missed monteggia fracture. J Pediatr Orthop 2017; 37: 20-22. 
20. Hung NN, Sang PC, Nam VT. Corrective ulnar osteotomy and annular ligament reconstruction by fascia lata in neglected monteggia fracture dislocation in children. International Journal of Orthopaedics 2017; 4(4): 802-81.

\begin{tabular}{|c|c|c|c|}
\hline \multicolumn{4}{|c|}{ AUTHORSHIP AND CONTRIBUTION DECLARATION } \\
\hline Sr. \# & Author(s) Full Name & Contribution to the paper & Author(s) Signature \\
\hline 1 & Abdul Latif Shahid & $\begin{array}{l}\text { Concept, Data collection, Study } \\
\text { design \& manuscript writing. }\end{array}$ & Shath \\
\hline 2 & Farhad Alam & Critical revising of study, Statistical & \\
\hline 3 & Islam Hussain & Proof reading. & \\
\hline 4 & Abdul Latif Sami & Supervision and Proof reading. & \\
\hline
\end{tabular}

